\title{
Enteric Myocarditis With Rhabdomyolysis Causing Acute Renal Failure: A Case Report
}

\author{
Authors \\ Chetan Jain ${ }^{1}$, Harshal Bhitkar ${ }^{2}$, Pramod Umarji ${ }^{3}$, Deelip Kadam ${ }^{4}$ \\ ${ }^{1}$ Medicine Resident, Department of Medicine BJMC Pune \\ Email: Chetanjain2312@gmail.com \\ ${ }^{2}$ Assistant Professor, Department of Medicine, BJMC Pune \\ ${ }^{3}$ Associate Professor, Department of Medicine, BJMC Pune \\ Email:mpuamrji@gmail.com \\ ${ }^{4}$ Professor and Head, Department of Medicine, BJMC Pune \\ Email:deelipkadam@gmail.com
}

\begin{abstract}
Rhabdomyolysis as well as myocarditis are considered to be rare complications of Salmonella infection. The diagnosis of these extra intestinal manifestations of enteric fever is often missed owing to the non specific symptoms. Life threatening complications of this condition make its prompt diagnosis and treatment imperative. Here we present case of a young male presenting with fever where diagnosis of enteric myocarditis with rhabdomyolysis and its prompt treatment helped in avoiding serious consequences. It serves to highlight the importance of clinical suspicion on part of the physician in providing timely diagnosis and effective treatment to the patient.
\end{abstract}

\section{Introduction}

The clinical presentation of Typhoid fever can be diverse with varied severity, thus making the clinical diagnosis of this febrile condition difficult. Complications occur in $10-15 \%$ of typhoid patients; gastrointestinal bleeding and intestinal perforation are most commonly involved. ${ }^{(1)}$.Rare complications include rhabdomyolysis, acute renal failure, pancreatitis, meningitis, myocarditis, pneumonia, arthritis, osteomyelitis and parotitis. (2)(3)(4) Myocarditis is a known complication of enteric fever that has almost classical ECG alterations. On the other hand, Rhabdomyolysis is not documented often, owing to varying severity of symptoms.It can however lead to acute renal failure, hence demanding that the physician should entertain diagnosis of infective aetiology for rhabdomyolysis. Although several organs can be affected in typhoid fever, the involvement of multiple organs in the same patient has rarely been reported in the literature.Here we present case of a young male presenting with fever where diagnosis of enteric myocarditis with rhabdomyolysis and its prompt treatment helped in avoiding serious consequences. The aim of presenting this case is to highlight the spectrum of multiple organ involvement in typhoid fever, which on rare occasions could occur simultaneously in the same patient. 


\section{Case Report}

A 34 year old male was referred to our hospital with history of fever for a week and chest pain for a day. Patient had severe generalised myalgia and intermittent left sided retrosternal chest pain. He did not have palpitation, syncope or sweating, there was no history of diarrhoea, cough with expectoration, rash or decreased urine output.Patient was a chronic alcoholic with no other significant past history. On general physical examination he appeared to have mild pallor and blood pressure of $96 / 70 \mathrm{mmHg}$. Auscultation revealed normal heart sounds and only occasional bilateral basal crepts.No skin rash was appreciated. Haematological laboratory findings and blood chemistry values on the day of admission and subsequent days are given in Table 1. Lab tests showed serum creatinine $7.2 \mathrm{mg} / \mathrm{dl}$ and blood urea $142 \mathrm{mg} \%$. Liver enzymes were within normal limits. The patient had metabolic acidosis with uric acid of 8.2. Serology for Dengue $\operatorname{IgM}$ and malarial antigen was negative. However HIV ELISA turned up positive with CD4 level of 257.ECG revealed $T$ wave inversion in $\mathrm{V} 2,3,4,5$ with ST changes and $1^{\text {st }}$ degree AV block (fig1). A day after admission patient developed breathlessness at rest. Pulse rate was $104 / \mathrm{min}$ and BP was 130/70 mmHg.On auscultation S3 became evident as did bilateral basal crepts. ECG showed Sinus Tachycardia and ST depression in v2 to v5. The creatinine kinase (CPK) level was significantly elevated at 1523 $\mathrm{U} / \mathrm{L}$ and CPKMB of 170 . Urine showed presence of myoglobin. (Day 2) Widal titres were: O - 1:80, $\mathrm{H}-1: 160$.Widal (repeat on day 6 of admission) $\mathrm{O}$ 1:160and H - 1:320. Blood culture showed Salmonella typhi sensitive to Ceftriaxone and ofloxacin and resistant to Amoxicillin. The patient initially was managed by intravenous Inj. Ceftriaxone $1 \mathrm{~g}$ bd which after positive Widal test was continued for 14 days. Inj Pantoprazole 40mg OD, Inj. Ondansetron 4mg tds and IV Fluids according to CVP were given.Good clinical response and complete normalization of most of the laboratory parameters were observed during treatment. On discharge, 14 days after admission, the patient was asymptomatic, enzyme levels had returned to normal, improvement in renal function and cardiac enzyme levels with treatment is shown in table 1. Definitive diagnosis of myocarditis requires endomyocardial biopsy, but in presence of evidence of blood culture, biochemical tests,ECG finding and transient nature of $\mathrm{CCF}$, diagnosis of Myocarditis with Rhabdomyolysis causing acute renal failure due to Enteric fever was made.Patient was discharged withTab ZLN bd and oral ciprofloxacin 500 bd as part of prophylaxis for 1 month along withTablet Pantoprazole.

\section{Discussion}

Typhoid fever is caused by virulent Gram negative bacilli called Salmonella typhi belonging to family Enterobacteriaceae.Humans are the only hosts.

Myocarditis occurs in 1-5\% patients of typhoid fever. It can mimic ischemia both electrographically and clinically. It is a precursor of DCM and CCF. Those patients with other systemic complications have a higher chance of having myocarditis.Proposed mechanisms of myocarditis include

- Humoral antibodies, either cytotoxic or interfering with contractile performance,

- Cellular immunity resulting in direct injury,

- Cytokines and growth factors. ${ }^{(5)}$

Typical ECG changes are often found in enteric myocarditis. Commonest ECG change is QT prolongation (29\%),ST-T changes (20\%),BBB (7\%) and AV blocks (1\%)(6).Presence of bizarre QRS and BBB is associated with bad prognosis. Treatmentconsists of diuretics and antibiotics along with supportive treatment may progress to DCM and CCF later .

Rhabdomyolysis with typhoid is very rare with only few cases reported till date. Muscle pain, elavated CPK total, myoglobin in urine with renal failure is hallmark.Salmonella induced rhabdomyolysis may be due to tissue 
hypoxia,toxins, direct bacterial invasion, low oxidative enzymes and activation of liposomal enzymes.Irrespective of the pathway finally increased calcium in myocyte is culprit (7).

Treatment of salmonella induced rhabdomyolysis includes hydration and antibiotics Sodium bicarbonate maintains alkaline urine that prevents conversion of myoglobin to ferriheamate, which is nephrotoxic. Hemodialysis may be required in some cases.

Salmonella species is known opportunistic infection in HIV infected patients, especially those not on treatment and with low CD4 count.Salmonella typhi can lead to bacteremia in $50 \%$ patients. It can lead to serious complications like meningitis, DIC, shock, endocarditis, myocarditis more often in HIV patients(8). Patients with complicated typhoid treated with ceftriaxone for 2 weeks have very high chance of recurrence. In such patients oral ciprofloxacin $500 \mathrm{bd}$ should be given as prophylaxis for as long as 6 months.

Life-threatening complications of this condition make its prompt diagnosis and treatment imperative.

\section{Conclusion}

The rarity of cardiac complications and muscle lysis due to typhoid fever may be deceptive. Salmonellosis causing multiple organ involvement has been reported even from developed countries where typhoid fever itself is a rarity. Electrocardiographic (ECG) alterations bear a direct relationship to the severity and prognosis of the disease process in myocarditis. It is important to look for infectious myocarditis in a patient who presents with fever and ECG abnormalities in the absence of other coronary risk factors. Rhadbdomyolysis is very rare but documented complication of salmonella causing acute renal failure. This case report is to remind the physicians regarding multiple organ involvement in salmonella infection.
Table1: Lab values of patient

\begin{tabular}{|l|l|l|l|l|l|l|}
\hline DAY & 1 & 3 & 5 & 7 & 9 & 12 \\
\hline UREA & 142 & 124 & 97 & 112 & 63 & 32 \\
\hline CREATININE & 7.2 & 6.8 & 5.7 & 4.2 & 3.1 & 2.0 \\
\hline CPK MB & 170 & & 97 & & & 40 \\
\hline
\end{tabular}

Fig 1 ECG s/o of prolonged PR interval with T wave inversion in $\mathrm{v} 2345$

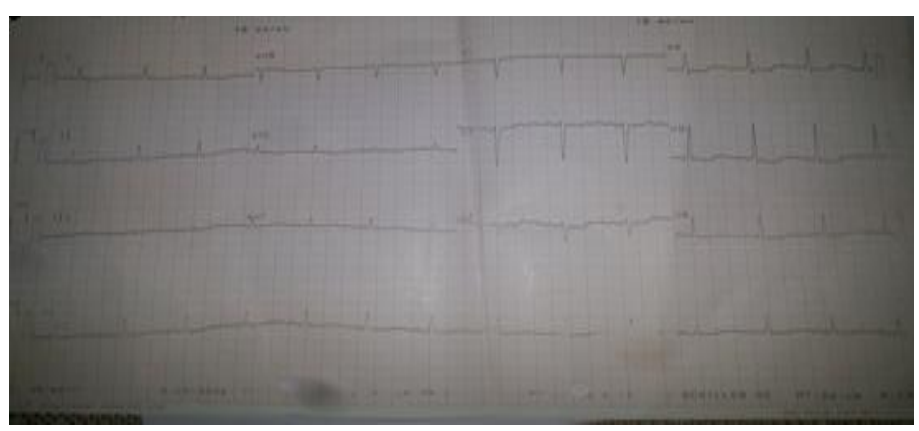

Fig 2 ECG s/o ST flattening in lateral leads

\section{References}

1. Parry CM, Hien TT, Dougan G, White NJ, Farrar JJ. Typhoid Fever. N Engl J Med. 2002;347(22):1770-82.

2. Khan FY, El-Hiday AH, Kamel HA. Typhoid osteomyelitis of the lumbar spine. Hong Kong Med J. 2006;12(5):391-3.

3. Chakraborty PP, Bhattacharjee R, Bandyopadhyay D. Complicated typhoid fever. J Assoc Physicians India. 2010; 58(3):186-7.

4. Shah S, Dubrey SW. Typhoid fever, complicated by myocarditis, in a traveller returning to the UK. BMJ Case Rep $2013 ; 2012-4$. 
5. Olinde KD, O'Connell JB. Inflammatory heart disease: pathogenesis, clinical manifestations, and treatment of myocarditis. Annu Rev Med. 1994;45:481-90.

6. Sathyamurthy I, Vidyalakshmi PR, Jayanthi K. Salmonella myocarditis presenting as acute myocardial infarction: a case report. Indian Hear $\mathbf{J}$

7. Brncic N, Viskovic I, Sasso A, Kraus I, Zamolo G. Salmonella infection-associated acute rhabdomyolysis. Some pathogenic considerations. Arch Med Res. 2002;33(3):313-5.

8. Grant AD, Djomand G, Smets P, Kadio A, Coulibaly M, Kakou A, et al. Profound immunosuppression across the spectrum of opportunistic disease among hospitalized HIV-infected adults in Abidjan, Cote d'Ivoire. AIDS. 1997;11(11):1357-64. 